\title{
Uncertainty and Sensitivity Studies with TRACE-SUSA and TRACE-DAKOTA by Means of Steady State BFBT Data
}

\author{
Wadim Jaeger, ${ }^{1}$ Victor Hugo Sánchez Espinoza, ${ }^{1}$ \\ Francisco Javier Montero Mayorga, ${ }^{2}$ and Cesar Queral $^{2}$ \\ ${ }^{1}$ Karlsruhe Institute of Technology, Institute for Neutron Physics and Reactor Technology, \\ Hermann-von-Helmholtz-Platz 1, 7634476344 Eggenstein-Leopoldshafen, Germany \\ ${ }^{2}$ Universidad Politecnica de Madrid, Departamento de Sistemas Energeticos ETSI Minas, \\ Alenza 4, 28003 Madrid, Spain
}

Correspondence should be addressed to Wadim Jaeger; wadim.jaeger@kit.edu

Received 16 October 2012; Accepted 8 February 2013

Academic Editor: Borut Mavko

Copyright (C) 2013 Wadim Jaeger et al. This is an open access article distributed under the Creative Commons Attribution License, which permits unrestricted use, distribution, and reproduction in any medium, provided the original work is properly cited.

\begin{abstract}
The subject of the present paper is the uncertainty and sensitivity studies for steady state BFBT results including pressure drop and void fraction measurements. The investigations are performed with TRACE (version 5.0 patch 2), thermal hydraulic modeling, and SUSA and DAKOTA; both tools are for the evaluation of uncertainties and sensitivities. For this purpose, the NUPEC BFBT experimental data base is used. The advantage of applying two different uncertainty and sensitivity tools in combination with TRACE is that the user effect can be excluded. Since in both cases the TRACE model of the BFBT bundle is identical the differences in the results are related to the capabilities of the uncertainty and sensitivity tools. The reference results with TRACE show that the code is very well able to represent both single- and two-phase flows even though it is a $1 \mathrm{D}$ coarse mesh system code. For selected cases, an uncertainty study was performed. Even though a reduced number of uncertain parameters are considered in the DAKOTA investigation, compared to the one with SUSA, similar results are obtained. The results indicate also that even small parameter variations can yield to rather large variations of the selected output parameters.
\end{abstract}

\section{Introduction}

The quality and reliability of the predictions of best-estimate system codes rely on the validation of their physical models. The present generation of system codes employs the latest physical models in order to describe the different phenomena which occur during normal and off-normal operation of Light Water Reactors (LWR). Due to the absence of mechanistic models empirical models are used to represent the underlying phenomena. Because of the complex nature of the involved physics, the limitations related to acquisition of accurate experimental data, for example, measurement fabrication, and the challenges related to computation and simulation of the models, but also the results, are afflicted by uncertainties. Since the current system codes are used as design and licensing tools of nuclear installations the uncertainties must be quantified in order to prove whether the investigated design stays within acceptance criteria and that physical limits are not exceeded. Input and boundary condition uncertainties are considered in this study.

Therefore, best-estimate codes and tools for the evaluation of uncertainties and the quantification of the sensitivities are applied together, called Best Estimate Plus Uncertainty (BEPU). In the present paper the system code TRACE is used to perform the thermohydraulic evaluation of a BWR fuel assembly which is used in the NUPEC BWR FullSize Fine Mesh Bundle Test (BFBT). For the uncertainty and sensitivity study the tool SUSA (Software system for Uncertainty and Sensitivity Analysis) [1-3] and the program DAKOTA (Design Analysis Kit for Optimization and Terascale Application) [4] are used. Both codes are used to perform the statistical propagation of input parameter uncertainties.

TRACE and other thermal hydraulic codes in combination with programs for uncertainty and sensitivity studies 
have been used since several years mainly for the analysis of experimental data and for licensing evaluation methodologies. The first methodology for uncertainty analysis applied to thermal hydraulic codes was the Code Scaling, Application, and Uncertainty methodology (CSAU), proposed by the US NRC. It is based on response surfaces for uncertainty treatment [5]. Later, other BEPU methodologies were developed replacing the response surface technique by statistical sampling methods. With these methodologies it is possible to determine the tolerance limit. Some of the most common methodologies in nuclear safety are ASTUM of Westinghouse, the SUSA package of the GRS, and TRACG of General Electrics. For further reading please refer to [6, 7]. Many international projects have been carried out in order to prove and to improve the existing methodologies including BEMUSE [8, 9], UAM [10]. The BEMUSE benchmark is a comparative exercise to, for example, evaluate the quality and applicability of best estimate system codes for uncertainty studies. While the BEMUSE phase is more related to best estimate codes like TRACE, CATHARE, or RELAP; the UAM benchmark is covering the different parts and levels of the whole calculation process: neutronics and thermal hydraulics multiscale and multiphysics application.

The NUPEC BFBT benchmark [11] is a source of experimental data of great variety for the purpose of code validation. In the frame of the BFBT test program, single- and twophase pressure losses, void fraction, and critical power tests were performed during steady state and transient conditions. In the present paper the pressure losses for single- and two-phase flow and the void fraction measurement data are selected for the comparative uncertainty and sensitivity study. For the investigations the same TRACE code version and the same input deck are used with SUSA and with DAKOTA. By doing so, the user effect is excluded from the analysis as well as the effect of different physical models since the same are always used. The authors are aware that even though user effect and physical model effect play no role, physical models may need improvement.

The propagation of uncertainties in model parameters is not considered in this study. The reasons are the missing information about the range of uncertainty of model parameters as well as their distribution. Currently, the international PREMIUM project endorsed by the OECD/NEA is aiming to evaluate the uncertainties of physical models or nonmeasureable parameters (e.g., interfacial drag coefficients, influence of bubble diameter). Based on the outcome of this project, physical parameter models can be included in such kind of investigation if their PDF and their range of variation are identified.

\section{BFBT Benchmark}

2.1. General Remarks. In the frame of the OECD/NEA NUPEC BFBT benchmark data have been provided related to pressure drop, critical power, and void fraction measurements for a BWR fuel assembly mock-ups. These data cover a wide range of BWR like operational conditions [11]. Stationary as well as transient results are provided covering not only normal operation but also typical BWR transients like a turbine trip without bypass or a trip of a recirculation pump. The experiments were focused $8 \times 8$ fuel assemblies. Two different designs have been investigated: a regular $8 \times 8$ fuel assembly with unheated rods in the center and a high burn-up assembly with a central water rod. In the experimental program, different scenarios characterized by different axial and radial power profiles were considered.

The benchmark is divided into two phases with the following sub topics.

(i) Phase I: void distribution benchmark

(a) Exercise 1: steady-state subchannel grade benchmark, where subchannel, meso- and microscopic approaches can be used.

(b) Exercise 2: steady-state microscopic grade benchmark, where meso- and microscopic approaches and molecular dynamics can be utilized.

(c) Exercise 3: transient macroscopic grade benchmark, where a subchannel approach can be applied.

(d) Exercise 4: uncertainty analysis of the void distribution benchmark.

(ii) Phase II: critical power benchmark

(a) Exercise 0: steady-state pressure drop benchmark.

(b) Exercise 1: steady-state benchmark, which applies a one-dimensional approach with BT correlations and a subchannel mechanistic approach.

(c) Exercise 2: transient benchmark, which applies a one-dimensional approach with BT correlations and a subchannel mechanistic approach.

The main features of the investigated fuel assembly, an $8 \times 8$ high burn up GE design, are summarized in Table 1 . Experiments were also performed for the other bundle types, but only the high burn-up version, assembly type 4 , is used for the present analysis.

2.2. Pressure Drop Measurements. The pressure drop has been measured during single and two phases where at single phase the pressure drop is the difference between the absolute pressures at two given positions minus the gravitational term. For two-phase flow condition, the pressure drop is simply the difference between the two considered elevations. The right-hand side of Figure 1 shows a sketch of the pressure sensor positions. PT N007 refers to the inlet of the heated section, while PTN010 is at the outlet of the heated section, $3708 \mathrm{~mm}$ above the inlet. At several positions pressure tabs are placed to account for, for example, the pressure drop over a spacer grid or the influence of rising elevations. For the present analysis, the pressure drop/difference between inlet and outlet is compared to the predictions. The differential pressure tab dpT9 is used for the comparison. 


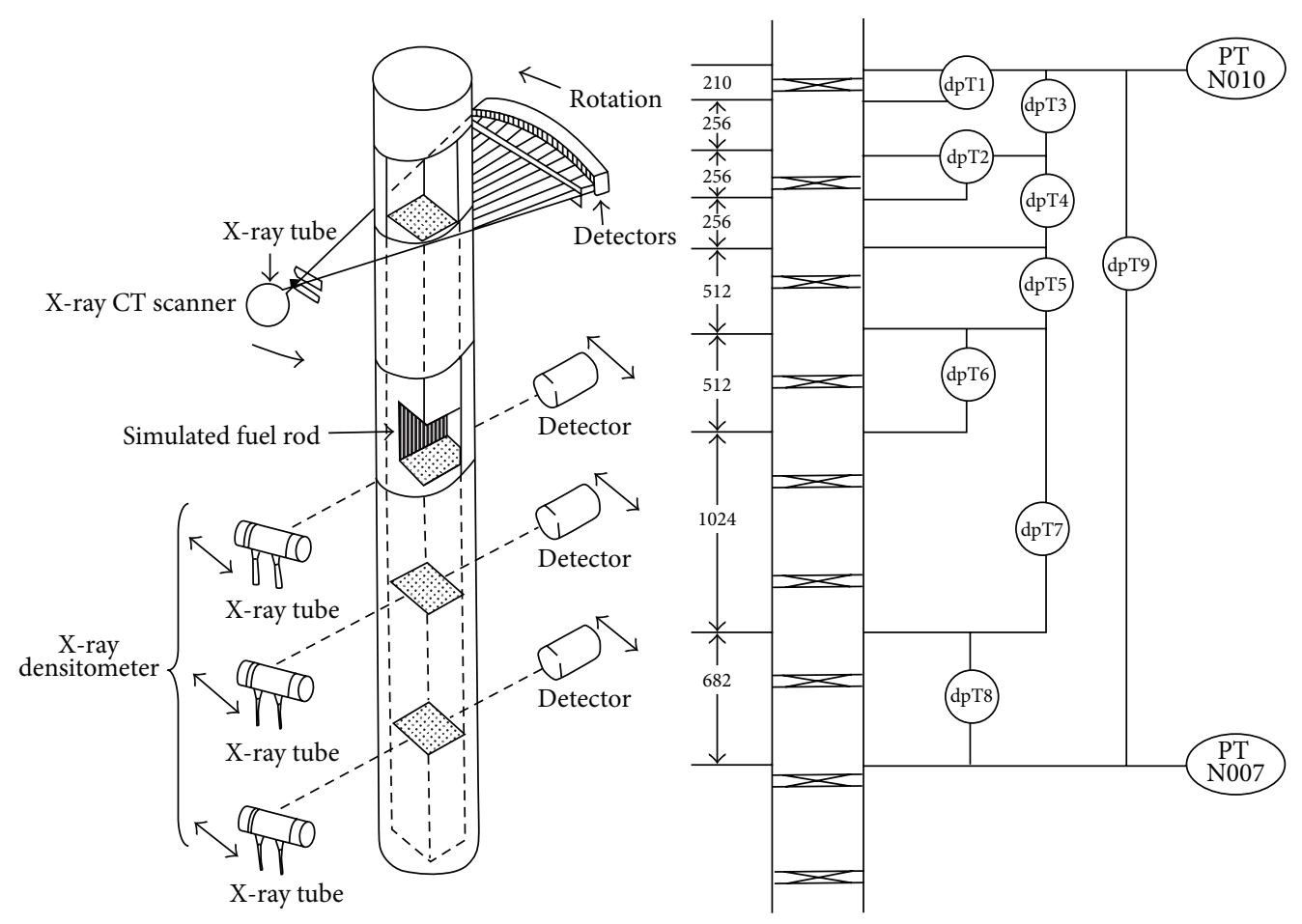

FIgURE 1: Void fraction and pressure drop measurement positions.

TABLE 1: Main features of assembly type 4.

\begin{tabular}{|c|c|}
\hline Parameter (unit) & Value \\
\hline Number of fuel rods (-) & 60 \\
\hline Outer diameter $(\mathrm{mm})$ & 12.3 \\
\hline Rod pitch (mm) & 16.2 \\
\hline Heated length (mm) & 3708 \\
\hline Number of water rods $(-)$ & 1 \\
\hline Channel box inner width (mm) & 132.5 \\
\hline Channel box corner radius ( $\mathrm{mm})$ & 8 \\
\hline In channel flow area $\left(\mathrm{mm}^{2}\right)$ & 9463 \\
\hline Number of spacers $(-)$ & 7 \\
\hline Spacer type $(-)$ & Ferrule \\
\hline Spacer form loss coefficient $(-)$ & 1.2 \\
\hline Spacer location (mm) & $\begin{array}{c}455,967,1479,1991,2503 \\
3015,3527\end{array}$ \\
\hline Heater outer diameter $(\mathrm{mm})$ & 7.3 \\
\hline Heater material $(-)$ & Nichrome \\
\hline Insulator outer diameter (mm) & 9.7 \\
\hline Insulator material (-) & Boron nitride \\
\hline Cladding thickness (mm) & 1.3 \\
\hline Cladding material (-) & Inconel 600/beryllium \\
\hline Axial power profile $(-)$ & uniform \\
\hline
\end{tabular}

In total, 36 combinations of pressure, inlet temperature, and mass flow rate for single-phase flow and 33 combinations of pressure, inlet subcooling, mass flow rate, and assembly power for two-phase flow are investigated during the experiments.

2.3. Void Fraction Measurements. The void fraction has been measured at four different elevations. The lower three elevations $(z=682 \mathrm{~mm}, 1706 \mathrm{~mm}$, and $2730 \mathrm{~mm}$ ) are equipped with $\mathrm{X}$-ray densitometers, while at the highest elevation $(z$ $=3758 \mathrm{~mm}$ ) an X-ray CT scanner was used, see left side of Figure 1. The X-ray densitometers could be moved in lateral direction and only a thin beam was penetrating the test section. With the X-ray CT scanner a more complete picture could be recorded since the detectors, in total 512, were rotating around the test section. The data measured with the X-ray densitometers are showing the void fraction of only a limited area of the subchannel since only between the rod rows the void fraction was captured. But the void fraction in the subchannel is not equally distributed as Glück pointed out [12]. At lower void fractions, for example, during bubbly flow, the void is concentrated in small bubbles close to the heat surface. At higher void fractions, for example, slug flow, bigger bubbles are located in the center of the subchannel. That means that the void fraction is underpredicted at lowvoid fractions and overpredicted at high-void fractions with the present X-ray densitometers. In [12] a corrective measure which writes as follows (with void in \%) is proposed:

$$
\alpha_{\text {corrected }}=\frac{\alpha_{\text {measured }}}{-0.001 \cdot \alpha_{\text {measured }}+1.167} \text {. }
$$

For the steady state cases, 86 combinations of pressure, inlet subcooling, mass flow rate, and power were considered. 


\section{Working Methodology}

3.1. TRACE-SUSA and TRACE-DAKOTA Interface. The TRACE code is a widely used best-estimate system code which is under development by US NRC and subcontractors. It is intended to be used for the analysis of LWRs and related facilities with the focus on normal, off-normal, and accidental scenarios. TRACE [13] features a 2-fluid, 6-equation approach where the conservation equations for mass, energy, and momentum are solved for the liquid and gas phase. Both phases have their own temperature and velocity field, while the pressure field is of common nature. Empirical closure laws and correlations are provided to calculate quantities like the wall-to-fluid heat transfer/drag or interfacial heat transfer/drag in order to close the conservative equations. Following a component approach, LWR typical components like valves, pumps, pipes, separators, and so forth can be modeled. For the present analysis, the CHAN component is used which has been especially developed for BWR fuel assemblies. The main feature of this component is that each pin can be represented with its respective power profile. In addition, water rods can be simulated.

TRACE is used for the thermohydraulic analysis of the BWR fuel assembly. The uncertainty and sensitivity study is performed with SUSA and DAKOTA. SUSA is the Software for Uncertainty and Sensitivity study developed by the GRS [3]. SUSA follows an input error propagation approach meaning that uncertainties of input parameters, for example, input and boundary conditions, material data or physical models, are taken into account by means of an uncertainty band and a distribution between the maximum and minimum value expressed by the probability density function (PDF). In addition, for the purpose of, for example, numerical stability analysis, nonphysical parameters like the axial or radial nodalization, or the time step size can be changed, too. These uncertainties propagate through the calculations and influence the output will have an uncertainty band. The input parameters are varied simultaneously within their minimum and maximum according to their assigned distribution. Each parameter is treated independently in case no correlation between them is present. In order to reach a chosen statistical fidelity, the number of samplings, being equal to the number of code runs, has to be settled prior to the analysis. The user has to define the probability content, $p$, which is the probability that a given variable will be between its lower and upper bounds and the level of confidence, $\beta$, which expresses that $p$ is overestimated conservatively with a certain probability meaning how confident one is that $p$ between the lower and upper bounds. The level of confidence takes into account the finite number of samplings/code runs. Accepted values for the probability content and the level of confidence are $95 \%$ for the two-sided tolerance limit. By means of quantiles, the Wilk's formula $[3,14]$ can be derived which connects the probability content and the level of confidence with the number of code running to be performed:

$$
\beta=1-p^{N}-N \cdot(1-p) \cdot p^{N-1} .
$$

Entering 0.95 for $p$ and $\beta$ will yield 93 for the number of code runs $(N)$. That means that 93 combinations of the input parameters will be considered for the uncertainty and sensitivity analysis. Figure 2 gives an overview about the information flow between TRACE and SUSA [15]. Both codes are run stand alone.

The input parameters with the uncertain range and the distribution are entered into SUSA. SUSA performs random sampling, as in the present study, or Latin hypercube sampling. A list with the chosen number of input parameter combinations will be generated.

By means of scripts the combinations are taken from SUSA and, for example, 93 new input decks are generated automatically. These 93 input decks will be executed and 93 outputs are generated. Information from these 93 outputs will be extracted with scripts and fed into SUSA to perform the actual uncertainty and sensitivity study. Since the data exchange is based on scripts any parameter in the input deck, or source code, can be considered in the study as long as all of the information (minimum, maximum, PDF, etc.) is known.

DAKOTA, developed by the Sandia National Laboratories, is described as a multilevel parallel object-oriented framework for design optimization, parameter estimation, uncertainty quantification, and sensitivity analysis [4]. DAKOTA has been implemented into the Symbolic Nuclear Analysis Package (SNAP) - the graphical user interface for codes like TRACE. The basic theory about sampling, uncertainty quantification, and sensitivity analysis is identical to SUSA. In addition, DAKOTA employs also the formula of Wilk. The data flow between TRACE and DAKOTA is similar to the one of TRACE and SUSA as depicted in Figure 2 with the exception that the data extracted is automatized and no actions from the user are required. Since everything is done in SNAP, the user does not need to write scripts for the data transfer. One only needs to select the input parameters, the uncertainty band with the corresponding distribution, the number of samples, the type of sampling, and the desired output parameters. The disadvantage of this process is that only parameters can be selected which are made available to DAKOTA in SNAP.

Currently, not all parameters of the input deck are available for uncertainty analysis, see the following section. Moreover, the parameters which can be selected are limited to the input deck. Model parameters cannot be selected yet even if all necessary uncertain parameters are known.

3.2. Selected Uncertain Parameters. For these investigations the output parameters of interest are the pressure drop (single and two phases) and the void fraction. The selected parameters are listed in Table 2 for TRACE-SUSA and TRACEDAKOTA along with information about the uncertainty range. All uncertainty parameters have in common that the probability density function will follow a normal distribution except for the inlet temperature which is flat (uniform). The range of the uncertainties is taken from the NUPEC specification [16]. Besides the inlet temperature all uncertainty ranges are given as percental variation.

The selection of the uncertain parameters is based, for example, on experience, expert judgment, the recommendations of the benchmark specifications, the access of these parameters (input or source code parameter), and whether 


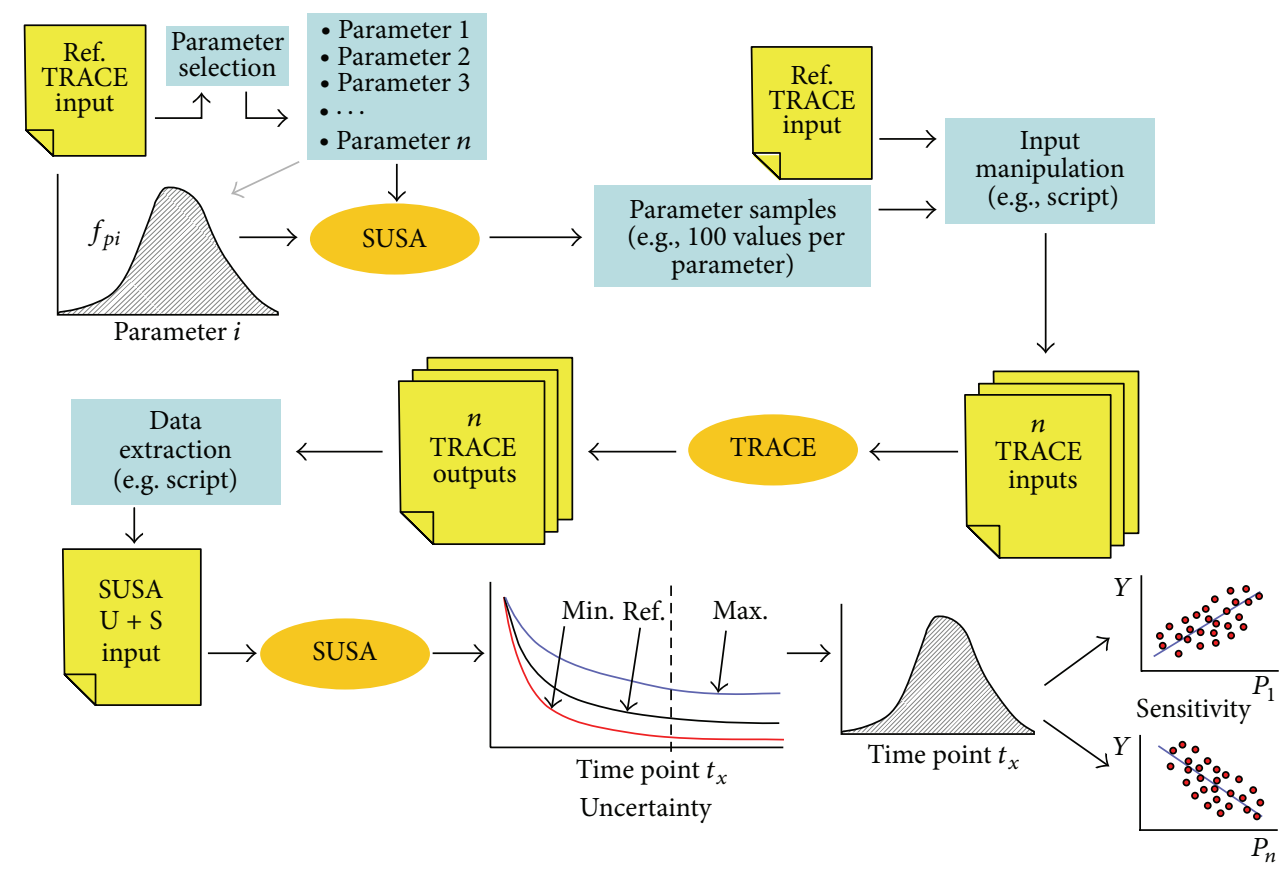

Figure 2: TRACE-SUSA information flow chart (with $N=100$ ).

TABLE 2: Uncertain parameters.

\begin{tabular}{lcccc}
\hline No. (SUSA/DAKOTA) & Parameter & Range & Distribution \\
\hline 1 & 1 & Outlet pressure & $\pm 1.0 \%$ & Normal \\
2 & 2 & Mass flow rate & $\pm 1.0 \%$ & Normal \\
3 & 3 & Inlet temperature & $\pm 1.5 \mathrm{~K}$ & Uniform \\
4 & 4 & Wall roughness & $\pm 5.0 \%$ & Normal \\
5 & 5 & K-spacer & $\pm 5.0 \%$ & Normal \\
6 & - & Hydraulic diameter & $\pm 1.0 \%$ & Normal \\
7 & - & Flow area & $\pm 1.0 \%$ & Normal \\
8 & 6 & Power & $\pm 1.5 \%$ & Normal \\
\hline
\end{tabular}

or not the uncertainties (minimal and maximal variation) are known. Furthermore, the selection has to consider the subject of the analysis. Since, for example, the pressure drops will be evaluating parameters like the mass flow rate, and the wall roughness is of interest. As the investigation will show, the same input parameters will have a different level of influence on the output parameters for the different investigations. In general, the physical connection between the input parameter and the output parameter must be given. Based in this relationship the influence is determined.

Besides the explicitly mentioned parameters like mass flow rate, power, inlet temperature/subcooling and hydraulic diameter, the parameters wall roughness and the form loss coefficient of the spacer grid have been considered, too. For the spacer grid a value of 1.2 is suggested by the benchmark team. An uncertainty of $\pm 5 \%$ is considered. For the wall roughness, not even a reference value is stated. Based on engineering judgment a value of $2.5 \mu \mathrm{m} \pm 5 \%$ is considered.
As mentioned in the previous subsection, not all parameters of the input deck can be selected and assigned with an uncertainty band. In addition, the capabilities related to the correlation between two parameters are limited. This is rather a problem of the DAKOTA implementation into SNAP than a problem with DAKOTA and SNAPS itself. Since DAKOTA has been implemented recently into SNAP, the full DAKOTA capabilities are not yet accessible, and the incorporation will also be improved in the near future. An example of the limited capabilities is the fact that the hydraulic diameter is not yet considered as uncertain parameter.

The TRACE model consists of 25 axial cells which must be all changed. With scripts it is an easy task to assign a multiplier to each cell. But with TRACE-DAKOTA every cell must be selected per hand requiring a lot of time to do such changes. The problem arises if parameters which are affected by the change of the hydraulic diameter must be changed, too. This is the case for the flow area and also for the volume. If the hydraulic diameter increases, the flow area increases, too. The hydraulic diameter is multiplied with, for example, a factor of 1.01, and the flow area is multiplied with a factor of $1.0201(1.01 \times 1.01)$ due to the quadratic dependency of the diameter. For the present range of the diameter uncertainty of $\pm 1.0 \%$ an almost perfect linear correlation is assumed between the multiplication factors for diameter and flow area. Thereby, the relation between the hydraulic diameter and the flow area is treated as for a cylindrical pipe. It was not possible to assign this dependency of the hydraulic diameter and the flow area with TRACE-DAKOTA. Therefore, the hydraulic diameter is not considered. Note that in the present investigation the hydraulic diameter and the flow area are the parameters which take into account the experimentally measured distortion of the assembly. That could be lateral 
TABLE 3: Input parameter combinations and uncertainty results for the single-phase flow pressure drop cases.

\begin{tabular}{|c|c|c|c|c|c|c|c|c|c|}
\hline Case (P700x) & Poutlet (bar) & $\operatorname{MFR}(\mathrm{t} / \mathrm{h})$ & Tinlet $(\mathrm{K})$ & Exp. (Pa) & Mean $(\mathrm{Pa})$ & $\operatorname{Max}(\mathrm{Pa})$ & $\operatorname{Min}(\mathrm{Pa})$ & $\mathrm{SD}(\mathrm{Pa})$ & Error (\%) \\
\hline \multirow{2}{*}{02} & \multirow{2}{*}{2.0} & \multirow{2}{*}{14.5} & \multirow{2}{*}{311.65} & \multirow{2}{*}{1920} & 1872 & 2010 & 1762 & 57 & -2.49 \\
\hline & & & & & 1881 & 1928 & 1831 & 24 & -2.05 \\
\hline \multirow{2}{*}{07} & \multirow{2}{*}{2.0} & \multirow{2}{*}{39.7} & \multirow{2}{*}{309.35} & \multirow{2}{*}{10990} & 10356 & 11266 & 9647 & 372 & -5.77 \\
\hline & & & & & 10371 & 10732 & 10009 & 181 & -5.63 \\
\hline \multirow{2}{*}{13} & \multirow{2}{*}{2.0} & \multirow{2}{*}{9.9} & \multirow{2}{*}{449.65} & \multirow{2}{*}{740} & 1004 & 1068 & 958 & 25 & 35.70 \\
\hline & & & & & 1010 & 1053 & 961 & 22 & 35.43 \\
\hline \multirow{2}{*}{16} & \multirow{2}{*}{9.8} & \multirow{2}{*}{24.8} & \multirow{2}{*}{449.35} & \multirow{2}{*}{4060} & 4252 & 4618 & 3988 & 144 & 4.73 \\
\hline & & & & & 4293 & 4427 & 4079 & 97 & 5.74 \\
\hline \multirow{2}{*}{30} & \multirow{2}{*}{71.6} & \multirow{2}{*}{34.7} & \multirow{2}{*}{558.85} & \multirow{2}{*}{8570} & 8975 & 9793 & 8385 & 318 & 4.73 \\
\hline & & & & & 9011 & 9366 & 8625 & 159 & 5.15 \\
\hline \multirow{2}{*}{35} & \multirow{2}{*}{71.6} & \multirow{2}{*}{64.8} & \multirow{2}{*}{557.75} & \multirow{2}{*}{22770} & 29588 & 32337 & 27604 & 1072 & 6.55 \\
\hline & & & & & 29657 & 30887 & 28221 & 605 & 6.80 \\
\hline
\end{tabular}

movement but also a reduced canister box width, a reduced pitch, or a changed rod diameter.

\section{Results}

In this section, the results obtained for single- and twophase flow pressure drop calculations as well as the void fraction calculation, are presented. First, the relative values obtained with respect to the experimental values are shown and followed by the evaluation of which uncertain parameters are the most important ones in each case for DAKOTA and SUSA calculations.

4.1. Single-Phase Flow Pressure Drop. All 36 scenarios for assembly type four have been modeled and simulated with TRACE. Six scenarios are selected for the uncertainty and sensitivity study. Meaning that 2 times (SUSA and DAKOTA) 6 times 93 equal to 1116 calculations are performed for this subsection. The results of the uncertainty analyses along with the input parameter combinations are given in Table 3 for SUSA and DAKOTA (the first values of mean, max, min, etc., are for SUSA, the second one for DAKOTA). The results, in relative form, are also plotted in Figure 3. One can see that, except for case P70013, the relative error is in the range of $\pm 5 \%$. For case P70013 an error of more than $35 \%$ has been calculated. A comparison of the absolute values shows that an experimental value of $740 \mathrm{~Pa}$ was measured, while the calculated one is in the range of $1000 \mathrm{~Pa}$. The absolute difference is only $260 \mathrm{~Pa}$ which is almost negligible. But the relative deviation is high. Figure 3 shows that the pressure drop is in general overpredicted, considering all 36 cases. One also can note that the uncertainty band for the TRACESUSA runs is wider than the one for TRACE-DAKOTA, as the smaller standard deviation indicates. That is related to the additional parameter, hydraulic diameter (flow area and volume) considered in the TRACE-SUSA but not in the TRACE-DAKOTA study.

In order to identify the input parameters where their variations have the largest influence on the single-phase flow

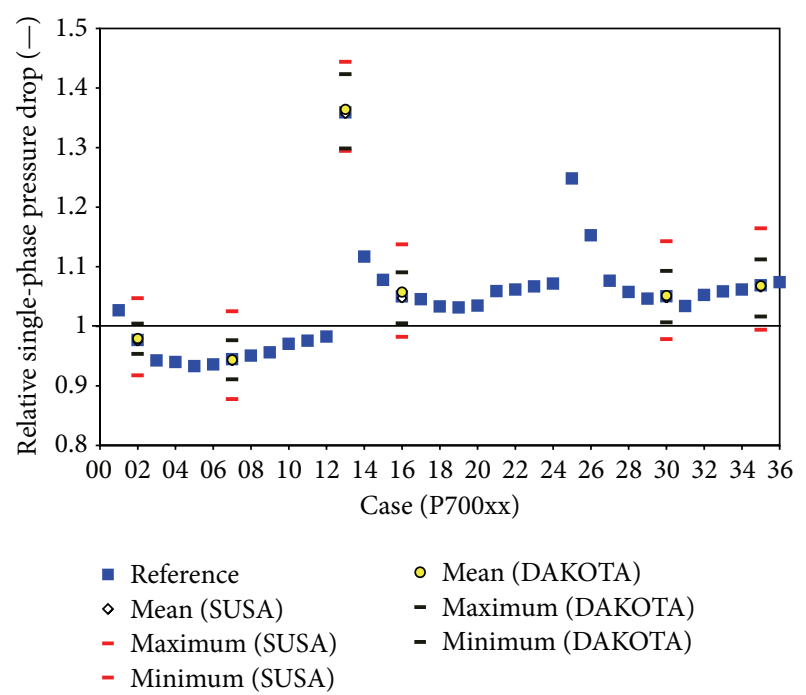

FIGURE 3: Relative single-phase flow pressure drop and uncertainty band for TRACE-SUSA and TRACE-DAKOTA.

pressure drop, a sensitivity analysis was performed. SUSA and DAKOTA use, among others, the Pearson's product momentum coefficient to quantify the correlation between input and output parameters. These coefficients are summarized in Table 4 (first line for SUSA, second for DAKOTA). With TRACE-SUSA, the hydraulic diameter is the most important parameter. A value of around -0.75 indicates a strong dependence, and a negative sign means that an increase of the hydraulic diameter results in a decrease of the pressure drop. Since the hydraulic diameter is not part of the TRACEDAKOTA analysis, a different parameter, the form loss coefficient of the spacers, is the dominant parameter. Outlet pressure, inlet temperature, and wall roughness show a weak or almost no dependency. In general, values between -0.3 and +0.3 indicate a weak dependency or an independency. The sensitivity coefficient of the mass flow rate is high for TRACEDAKOTA but low for TRACE-SUSA. Since the hydraulic 
TABLE 4: Sensitivity coefficients for the single-phase flow pressure drop.

\begin{tabular}{lcccccc}
\hline Case (P700x) & Outlet pressure & Mass flow rate & Inlet temperature & Wall roughness & K-spacer & Hydraulic diameter \\
\hline \multirow{2}{*}{02} & 0.143 & 0.281 & -0.240 & -0.106 & 0.539 & -0.749 \\
& 0.030 & 0.550 & 0.045 & 0.076 & 0.836 & - \\
07 & 0.152 & 0.283 & -0.229 & -0.131 & 0.570 & -0.747 \\
& 0.007 & 0.506 & 0.003 & -0.008 & 0.859 & - \\
13 & 0.138 & 0.287 & -0.184 & -0.081 & 0.585 & -0.754 \\
& 0.045 & 0.636 & -0.113 & -0.029 & 0.764 & - \\
16 & 0.150 & 0.285 & -0.174 & -0.093 & 0.603 & -0.751 \\
& 0.044 & 0.851 & -0.200 & -0.022 & 0.476 & -0.614 \\
30 & 0.154 & 0.284 & -0.135 & -0.108 & -0.749 \\
35 & -0.022 & 0.510 & -0.062 & 0.024 & 0.849 & - \\
& 0.152 & 0.282 & -0.129 & -0.112 & 0.624 & -0.747 \\
\hline
\end{tabular}

TABLE 5: Input parameter combinations and uncertainty results for the two-phase flow pressure drop cases.

\begin{tabular}{|c|c|c|c|c|c|c|c|c|c|c|}
\hline Case (P600x) & Poutlet (bar) & $\operatorname{MFR}(\mathrm{t} / \mathrm{h})$ & $\begin{array}{c}\text { hsub } \\
(\mathrm{kJ} / \mathrm{kg})\end{array}$ & $P(\mathrm{MW})$ & Exp. $(\mathrm{Pa})$ & Mean $(\mathrm{Pa})$ & $\operatorname{Max}(\mathrm{Pa})$ & $\operatorname{Min}(\mathrm{Pa})$ & $\mathrm{SD}(\mathrm{Pa})$ & Error (\%) \\
\hline \multirow{2}{*}{01} & \multirow{2}{*}{71.6} & \multirow{2}{*}{20.2} & \multirow{2}{*}{53.3} & \multirow{2}{*}{0.863} & \multirow{2}{*}{27400} & 26376 & 26955 & 25788 & 264 & -3.74 \\
\hline & & & & & & 26369 & 26840 & 25915 & 196 & -3.76 \\
\hline \multirow{2}{*}{08} & \multirow{2}{*}{71.7} & \multirow{2}{*}{55.0} & \multirow{2}{*}{49.0} & \multirow{2}{*}{3.057} & \multirow{2}{*}{65720} & 64000 & 68566 & 60755 & 1732 & -2.62 \\
\hline & & & & & & 63774 & 66553 & 61297 & 1098 & -2.96 \\
\hline \multirow{2}{*}{14} & \multirow{2}{*}{71.6} & \multirow{2}{*}{70.1} & \multirow{2}{*}{50.6} & \multirow{2}{*}{3.884} & \multirow{2}{*}{91270} & 90818 & 98174 & 85491 & 2817 & -0.50 \\
\hline & & & & & & 90257 & 93822 & 86191 & 1835 & -1.11 \\
\hline \multirow{2}{*}{20} & \multirow{2}{*}{71.6} & \multirow{2}{*}{45.1} & \multirow{2}{*}{51.8} & \multirow{2}{*}{4.363} & \multirow{2}{*}{68830} & 64003 & 68386 & 60185 & 1777 & -7.01 \\
\hline & & & & & & 63712 & 65725 & 61955 & 960 & -7.44 \\
\hline \multirow{2}{*}{22} & \multirow{2}{*}{86.4} & \multirow{2}{*}{20.2} & \multirow{2}{*}{50.7} & \multirow{2}{*}{0.837} & \multirow{2}{*}{26830} & 26008 & 26587 & 25435 & 262 & -3.06 \\
\hline & & & & & & 25934 & 26492 & 25377 & 236 & -3.34 \\
\hline \multirow{2}{*}{30} & \multirow{2}{*}{86.4} & \multirow{2}{*}{70.2} & \multirow{2}{*}{51.4} & \multirow{2}{*}{5.076} & \multirow{2}{*}{100770} & 101399 & 109640 & 95273 & 3216 & 0.62 \\
\hline & & & & & & 100810 & 105480 & 95578 & 2086 & 0.04 \\
\hline
\end{tabular}

diameter is the major source for the pressure drop variation the sensitivity coefficient of the mass flow rate, and of all other parameters, must be higher if that parameter is not present in the analysis. Based on the present result the ranking is as follows: $1=$ hydraulic diameter, $2=K$-spacer, and $3=$ mass flow rate.

4.2. Two-Phase Flow Pressure Drop. As for the single-phase case, all scenarios for assembly type four are modeled, and 6 representative scenarios are selected for the uncertainty and sensitivity analysis. The input and boundary parameter combinations of the six selected cases are given on the left side of Table 5. The right side shows the result of the uncertainty analysis, and Figure 4 shows the mean, maximum, and minimum for SUSA and DAKOTA in relative values. It can be seen that the calculations underpredict the experimental results. The overall error is in the order of $\pm 5 \%$. For the six selected cases the absolute error ranges from almost zero to $7.5 \%$. Again, the TRACE-DAKOTA results are characterized by the smaller standard deviation and the resulting narrower uncertainty band.

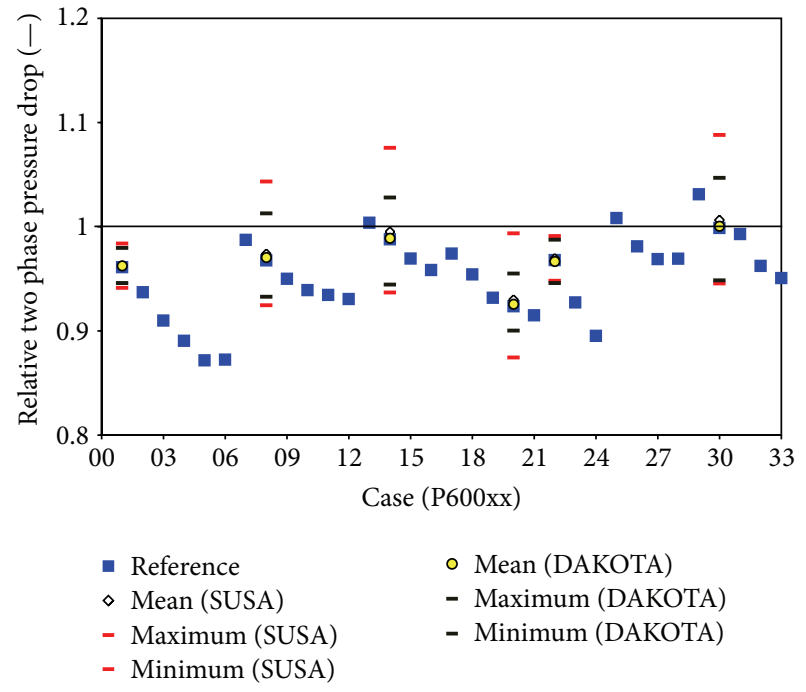

FIgURE 4: Relative two-phase flow pressure drop and uncertainty band for TRACE-SUSA and TRACE-DAKOTA. 
TABLE 6: Sensitivity coefficients for the two-phase flow pressure drop.

\begin{tabular}{|c|c|c|c|c|c|c|c|}
\hline Case (P600x) & Outlet pressure & Mass flow rate & Inlet temperature & Wall roughness & $K$-spacer & Hydraulic diameter & Power \\
\hline \multirow{2}{*}{01} & 0.189 & 0.212 & -0.645 & -0.013 & 0.387 & -0.486 & -0.040 \\
\hline & -0.391 & 0.322 & 0.802 & 0.314 & 0.002 & - & 0.029 \\
\hline \multirow{2}{*}{08} & -0.184 & 0.263 & 0.406 & 0.055 & 0.342 & -0.700 & 0.126 \\
\hline & -0.396 & 0.287 & 0.672 & 0.087 & 0.509 & - & 0.013 \\
\hline \multirow{2}{*}{14} & -0.197 & 0.252 & 0.447 & 0.058 & 0.328 & -0.678 & 0.131 \\
\hline & -0.394 & 0.272 & 0.730 & 0.061 & 0.408 & - & 0.232 \\
\hline \multirow{2}{*}{20} & -0.158 & 0.245 & 0.308 & 0.044 & 0.331 & -0.750 & 0.181 \\
\hline & -0.413 & 0.284 & 0.641 & 0.086 & 0.485 & - & 0.334 \\
\hline \multirow{2}{*}{22} & 0.202 & 0.203 & -0.653 & -0.017 & 0.390 & -0.479 & -0.033 \\
\hline & -0.280 & 0.250 & 0.865 & 0.030 & 0.263 & - & 0.098 \\
\hline \multirow{2}{*}{30} & -0.193 & 0.250 & 0.400 & 0.056 & 0.338 & -0.701 & 0.149 \\
\hline & -0.445 & 0.269 & 0.646 & 0.070 & 0.502 & - & 0.294 \\
\hline
\end{tabular}

TABLE 7: Input parameter combinations and uncertainty results for the void fraction cases.

\begin{tabular}{|c|c|c|c|c|c|c|c|c|c|c|}
\hline Case (4101-) & Poutlet (bar) & $\operatorname{MFR}(\mathrm{t} / \mathrm{h})$ & $\begin{array}{c}\text { hsub } \\
(\mathrm{kJ} / \mathrm{kg})\end{array}$ & $P(\mathrm{MW})$ & Exp. $(\mathrm{Pa})$ & Mean (-) & $\operatorname{Max}(-)$ & $\operatorname{Min}(-)$ & $\mathrm{SD}(-)$ & Error (\%) \\
\hline \multirow{2}{*}{04} & \multirow{2}{*}{9.73} & \multirow{2}{*}{10.2} & \multirow{2}{*}{43.9} & \multirow{2}{*}{0.82} & \multirow{2}{*}{0.840} & 0.843 & 0.847 & 0.838 & 0.002 & 0.31 \\
\hline & & & & & & 0.842 & 0.846 & 0.838 & 0.002 & 0.25 \\
\hline \multirow{2}{*}{18} & \multirow{2}{*}{39.19} & \multirow{2}{*}{10.14} & \multirow{2}{*}{51.5} & \multirow{2}{*}{1.00} & \multirow{2}{*}{0.755} & 0.778 & 0.785 & 0.770 & 0.003 & 2.98 \\
\hline & & & & & & 0.779 & 0.785 & 0.770 & 0.003 & 3.14 \\
\hline \multirow{2}{*}{29} & \multirow{2}{*}{39.17} & \multirow{2}{*}{54.68} & \multirow{2}{*}{50.3} & \multirow{2}{*}{3.92} & \multirow{2}{*}{0.757} & 0.766 & 0.773 & 0.758 & 0.004 & 1.17 \\
\hline & & & & & & 0.765 & 0.774 & 0.757 & 0.004 & 1.11 \\
\hline \multirow{2}{*}{47} & \multirow{2}{*}{71.32} & \multirow{2}{*}{29.94} & \multirow{2}{*}{50.6} & \multirow{2}{*}{1.43} & \multirow{2}{*}{0.536} & 0.573 & 0.594 & 0.551 & 0.010 & 6.97 \\
\hline & & & & & & 0.573 & 0.599 & 0.552 & 0.010 & 6.97 \\
\hline \multirow{2}{*}{64} & \multirow{2}{*}{71.59} & \multirow{2}{*}{69.53} & \multirow{2}{*}{53.6} & \multirow{2}{*}{1.57} & \multirow{2}{*}{0.263} & 0.254 & 0.327 & 0.181 & 0.033 & 3.38 \\
\hline & & & & & & 0.254 & 0.312 & 0.186 & 0.033 & 3.38 \\
\hline \multirow{2}{*}{82} & \multirow{2}{*}{86.35} & \multirow{2}{*}{54.54} & \multirow{2}{*}{52.9} & \multirow{2}{*}{1.85} & \multirow{2}{*}{0.376} & 0.416 & 0.456 & 0.375 & 0.018 & 10.54 \\
\hline & & & & & & 0.415 & 0.450 & 0.377 & 0.020 & 10.31 \\
\hline
\end{tabular}

The sensitivity coefficients have almost similar values for all considered cases, see Table 6 . The fluctuations are related to the varying input and boundary conditions. The only exception is the inlet temperature which has relative high negative values for cases 1 and 22. But for the rest of the cases positive values between $0.3-0.4$ are calculated. For the named cases, the boundary conditions show that they are characterized by low mass flow rate and low power. Therefore, the impact of their variation is losing against the impact of the inlet subcooling (inlet temperature) which is almost constant for all cases. The highest values are calculated for the hydraulic diameter followed by the inlet temperature and the $K$-factor for the spacer grid.

The sensitivity coefficients of the TRACE-DAKOTA analysis indicate a slightly different relation between the parameters. For the inlet temperature exclusively high positive values, $>0.6$, are calculated, even for case 1 and 22 for which the TRACE-SUSA analysis predicted negative values of 0.65 . That means that for the TRACE-SUSA analysis the pressure drop would increase with decreasing inlet temperature. For TRACE-DAKOTA the influence of the inlet temperature would be in the opposite direction; increasing inlet temperature yields increasing pressure drop. One explanation could be the absence of the hydraulic diameter, which amplifies the influence of the inlet temperature compared to the TRACESUSA analysis.

4.3. Void Fraction. Six cases out of 86 are selected for this investigation. The input and boundary conditions and the results regarding the uncertainties are given in Table 7 . Considering the reference results of all 86 scenarios, a tendency to overpredict the void fraction can be seen. In addition, the relative error is slightly depending on the void fraction. At higher void fractions the error is lower than at lower void fractions. Another finding is that the uncertainty band is wider at lower void fractions as shown in Table 7 and in Figure 5. At a void fraction of 0.84 , as in case 410104 , the uncertainty band can be almost neglected, while for case 410164 , void $=0.263$, the band is clearly visible.

For two of the six cases the void fraction is plotted as function of the axial height in Figure 6 and Figure 7 for case 410104 and 410164, respectively. The same tendency 


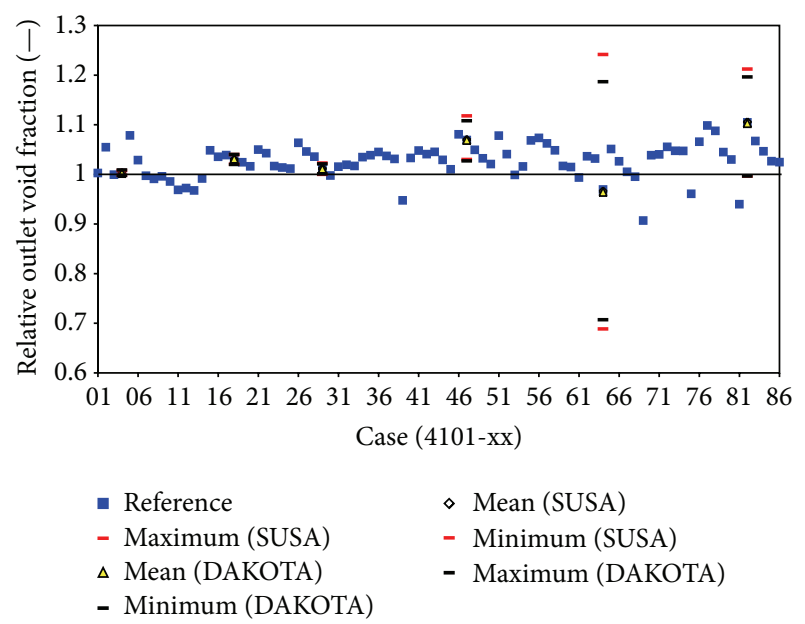

FIGURE 5: Relative outlet void fraction and uncertainty band for TRACE-SUSA and TRACE-DAKOTA.
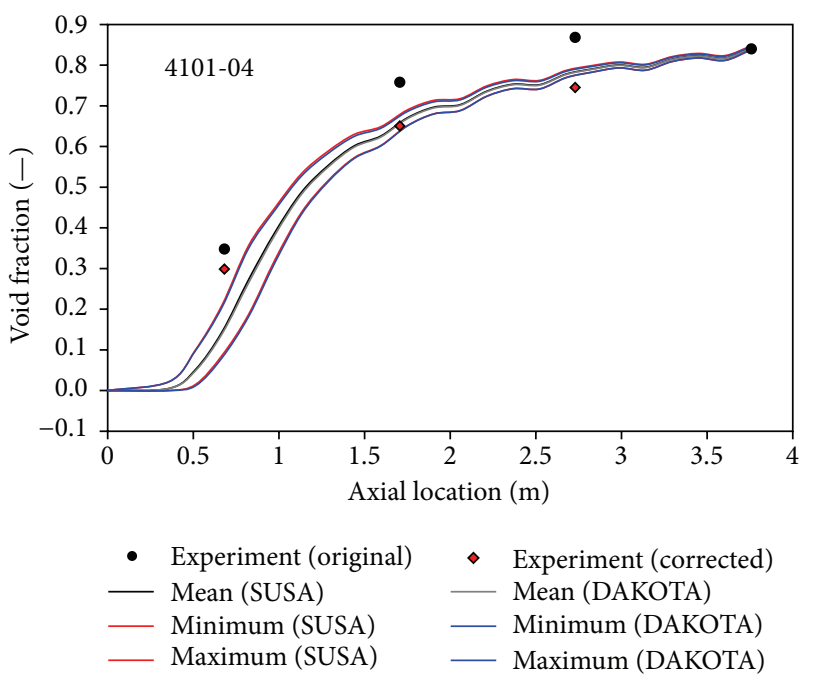

FIgURE 6: Axial void fraction profile for case 4101-04.

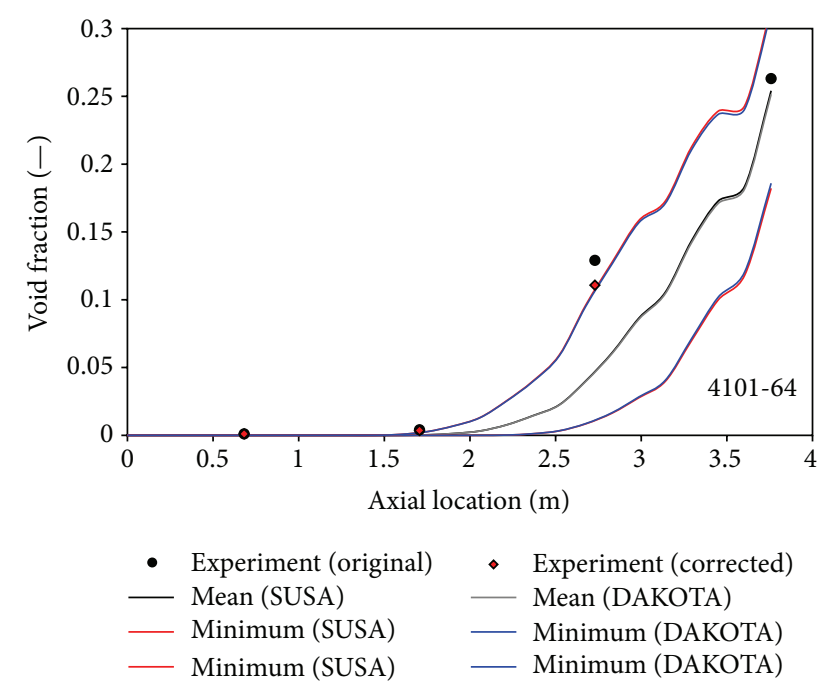

Figure 7: Axial void fraction profile for case 4101-64. can be seen. The higher the void fraction the closer the minimum and maximum are to the mean value. In addition to the corrected results, the original measured data points are plotted. It can be seen that the correction is more pronounced at higher void fractions. It is also clearly visible that considering the original void fractions, the TRACE predictions will always underpredict the measurements. With the corrections, the discrepancies are reduced. With both uncertainty codes almost identical results are obtained. That means that the hydraulic diameter, as the only difference between the two codes, is not important as it is for the pressure drop simulations. That can be best seen by the identical standard deviations.

The wider uncertainty band at lower void fractions might be related to the flow regime which is present at the different void fractions. At lower void fractions the regime is bubbly slug flow, while at higher void fractions annular mist flow. The differences in calculating the interfacial friction and their sensitivity to the parameters which are varied could be one reason for the observed behavior.

The sensitivity coefficients according to Pearson's momentum correlation coefficient are given in Table 8. It is clearly visible that the inlet temperature, which in fact represents the inlet subcooling, is the input parameter of highest importance. Besides the inlet temperature, the power is also of importance, but the range of the sensitivity coefficient ranges from almost zero to values up to 0.6. At low-void fractions, the values for the mass flow rate are close to zero while for high-void fraction the values are close to -0.25 , meaning that an increase of the mass flow rate always results in a decrease of the void fraction. Parameters like the hydraulic diameter or $K$ factor have almost no impact on the outlet void fraction which explains why TRACE-SUSA and TRACE-DAKOTA predict the same results. It can be seen that at low-void fractions the sensitivity coefficient for the inlet temperature is very high, around 0.9 , while the one for the power is very low. With increasing void fraction the sensitivity coefficient for the inlet temperature decreases. The coefficient for the power is increasing linearly with increasing void fractions, going from $<0.1$ at a void fraction of 0.25 to values of around 0.6 at void fractions $>0.8$. Since the variation is almost constant, $\pm 1.5 \mathrm{~K}$ for the inlet temperature and $\pm 1 \%$ for the power, the change of the absolute values is getting more pronounced with higher temperatures and powers. With increasing assembly power the outlet void fraction is increased. That means that a variation of $1 \%$ of an already high assembly power will result in much more additional energy which is released in the systems as $1 \%$ at a low assembly power. At low-void fractions, the variation of the inlet subcooling (inlet temperature) has more influence on the resulting void fraction as at higher void fractions because the inlet subcooling is the only parameter which affects the results according to the sensitivity study.

\section{Summary and Conclusions}

In this paper steady state results of the NUPEC BFBT data base for single- and two-phase flow pressure drop and void 
TABLE 8: Sensitivity coefficients for the void fraction cases.

\begin{tabular}{|c|c|c|c|c|c|c|c|}
\hline Case (4101-) & Outlet pressure & Mass flow rate & Inlet temperature & Wall roughness & $K$-spacer & Hydraulic diameter & Power \\
\hline \multirow{2}{*}{04} & -0.159 & -0.216 & 0.721 & -0.072 & -0.150 & -0.205 & 0.461 \\
\hline & -0.474 & -0.239 & 0.640 & -0.052 & -0.012 & - & 0.500 \\
\hline \multirow{2}{*}{18} & -0.225 & -0.205 & 0.651 & -0.071 & -0.120 & -0.275 & 0.500 \\
\hline & -0.415 & -0.246 & 0.656 & 0.029 & 0.012 & - & 0.567 \\
\hline \multirow{2}{*}{29} & -0.250 & -0.252 & 0.718 & -0.060 & -0.210 & 0.043 & 0.352 \\
\hline & -0.476 & -0.236 & 0.698 & -0.039 & 0.005 & - & 0.459 \\
\hline \multirow{2}{*}{47} & -0.331 & -0.114 & 0.830 & 0.021 & -0.167 & -0.024 & 0.213 \\
\hline & -0.425 & -0.185 & 0.828 & 0.010 & 0.014 & - & 0.284 \\
\hline \multirow{2}{*}{64} & -0.304 & -0.018 & 0.899 & 0.053 & -0.162 & 0.004 & 0.053 \\
\hline & -0.450 & -0.080 & 0.884 & -0.006 & 0.001 & - & 0.098 \\
\hline \multirow{2}{*}{82} & -0.343 & -0.059 & 0.864 & 0.045 & -0.167 & 0.011 & 0.115 \\
\hline & -0.502 & -0.130 & 0.862 & -0.016 & 0.022 & - & 0.180 \\
\hline
\end{tabular}

fraction measurements are used to perform an uncertainty and sensitivity study with TRACE-SUSA and TRACEDAKOTA. One major drawback of the current TRACEDAKOTA tool is that not all input and boundary conditions can be included due to programming or practical reason. That becomes noticeable when inclusion of the geometrical parameters is needed.

Especially for the calculation of the pressure drop, the variation of the hydraulic diameter is important. In fact, for the single-phase flow pressure drop analysis it turned out that this parameter is the most important one in terms of sensitivity related to its variation. For the two-phase flow cases the importance changed. In this case, the inlet temperature, which is taking into account the inlet subcooling, is the dominant parameter, while the hydraulic diameter and also the form loss coefficient of the spacer grid are of less importance. That is reasonable since the change of the inlet temperature will provoke a change of the void fraction.

Concerning the void fraction cases, TRACE-SUSA and TRACE-DAKOTA predict similar results. This means that the variation of the hydraulic diameter is not important. This has also been proofed by the calculated sensitivity coefficients which are similar for both codes. Besides the general insignificance of the hydraulic diameter in the present case, a reason might be also the rather small variation of the parameter. A larger, for example, 5\%, variation may cause differences for both options. One important finding of that investigation is that the width of the uncertainty band is a function of the void fraction. At high-void fractions the influence of the inlet subcooling is minimal, while the one for the power is gaining importance. At low-void fractions, the investigation showed that only the inlet temperature has an influence, while the power can be declared unimportant.

In general, the comparison between measurements and predictions shows a good agreement which proofs that TRACE is able to represent the stationary behavior in a BWR fuel assembly at different operational conditions.

Based on the present investigation it can be concluded that both tools TRACE-SUSA and TRACE-DAKOTA give similar results if the same parameters are included in the investigation. That is comprehensible since SUSA and
DAKOTA are based on the same mathematical formulations. From the practical point of view DAKOTA has the advantage that it is implemented in SNAP and the user can use the same graphical interface as with TRACE. In addition, programming skills are not required since everything is automatized. The major drawback of DAKOTA is that in its current implementation stage as it cannot be used if parameters are correlated to each other. In case the investigation is focused on physical model parameters, DAKOTA cannot be used at all. This is the biggest advantage of SUSA. Even though programming skills are mandatory, the user can change every parameter which needs to be considered in the uncertainty and sensitivity analysis.

\section{Conflict of Interests}

The authors hereby declare that no conflict of interest is present between them and the commercial entities mentioned in the context of the paper.

\section{Acknowledgments}

Thanks are due to the Program "Nuclear Safety Research" of KIT for the financial support of the Research Topic "Multiphysics Methodologies for Reactor Dynamics and Safety" and to the Deutscher Akademischer Austausch Dienst (DAAD).

\section{References}

[1] B. Krzykacs, E. Hofer, and M. Kloos, "A software system for probabilistic uncertainty and sensitivity analysis of results from computer models," Proceedings of the International Conference on Probabilistic Safety Assessment and Management, San Diego, USA, 1994.

[2] GRS, SUSA V3.5 user's guide and tutorial, Gesellschaft fuer Anlagen- und Reaktorsicherheit, Cologne, Germany, 2002.

[3] H. Glaeser, "GRS method for uncertainty and sensitivity evaluation of code results and applications," Science and Technology of Nuclear Installations, vol. 2008, Article ID 798901, 2008.

[4] B. Adams, K. Dalbey, M. Eldred, D. Gay, L. Swiler, W. Bohnhoff et al., DAKOTA, A Multilevel Parallel Object-Oriented 
Framework for Design Optimization, Parameter Estimation, Uncertainty Quantification, and Sensitivity Analysis Version 5.1 User's Manual, Sandia National Laboratories, Livermore, Calif, USA, 2011.

[5] B. E. Boyack, I. Catton, R. B. Duffey et al., "Quantifying reactor safety margins part 1: an overview of the code scaling, applicability, and uncertainty evaluation methodology," Nuclear Engineering and Design, vol. 119, no. 1, pp. 1-15, 1990.

[6] M. Nissley, C. Frepoli, K. Ohkawa, and K. Muftuoglu, Realistic Large-Break LOCA Evaluation Methodology Using the Automated Statistical Treatment of Uncertainty Method (ASTRUM). WCAP-16009-NP, Westinghouse Electric Company, Pittsburgh, $\mathrm{Pa}, \mathrm{USA}, 2003$.

[7] S. Kawamura and T. Hara, Best Estimate Methods for Licensing Analysis. Best-Estimate Methods in Nuclear Installation Safety Analysis, American Nuclear Society, Washington, DC, USA, 2000.

[8] A. de Crécy, P. Bazin, H. Glaeser, T. Skorek, J. Joucla, P. Probst et al., "BEMUSE phase III report: Uncertainty and sensitivity analysis of the LOFT L2-5 test," Tech. Rep. NEA/CSNI/R(2007)4, 2007.

[9] F. Reventos, M. Perez, L. Batet, A. Guba, I. Tóth, T. Mieusset et al., "BEMUSE phase V report: Uncertainty and sensitivity analysis of a LBLOCA in Zion nuclear power plant," Tech. Rep. NEA/SCNI/R(2009)13, 2009.

[10] K. Ivanov, M. Avramova, I. Kodeli, and E. Sartori, Benchmark for Uncertainty Analysis in Modelling (UAM) for Design, Operation and Safety Analysis of LWRs Volume I: Specification and Support Data for the Neutronics Cases, OECD Nuclear Energy Agency, 2007.

[11] B. Neykov, F. Aydogan, L. Hochreiter, K. Ivanov, H. Utsuno, K. Fumio et al., NUPEC BWR Full-Size Fine Mesh Buncle Test (BFBT) Benchmark Volume I: Specifications, OECD/NEA, 2005.

[12] M. Glück, "Validation of the sub-channel code F-COBRATF. Part II. Recalculation of void measurements," Nuclear Engineering and Design, vol. 238, no. 9, pp. 2317-2327, 2008.

[13] US NRC, TRACE V5. 0 Theory Manual-Field Equations, Solution Methods, and Physical Models, U.S. Nuclear Regulatory Commission, 2010.

[14] S. Wilks, "Determination of sample sizes for setting tolerance limits," The Annals of Mathematical Statistics, vol. 12, no. 1, pp. 91-96, 1941.

[15] W. Jäger, V. Sánchez, and R. Macián-Juan, “On the uncertainty and sensitivity analysis of experiments with supercritical water with TRACE and SUSA," Proceedings of the 18th International Conference on Nuclear Enginnering (ICONE '10), Xian, China, 2010.

[16] B. Neykov, L. Hochreiter, K. Ivanov, H. Utsuno, E. Sartori, and M. Martin, NUPEC BWR Full-Size Fine-Mesh Bundle Test (BFBT Benchmark) Volume II: Uncertainty and Sensitivity Analyses of Void Distribution and Critical Power-Specification, OECD Nuclear Energy Agency, 2007. 


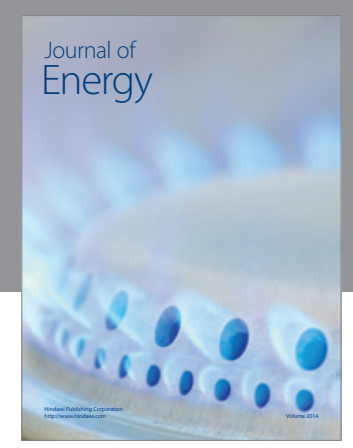

Journal of

Industrial Engineering
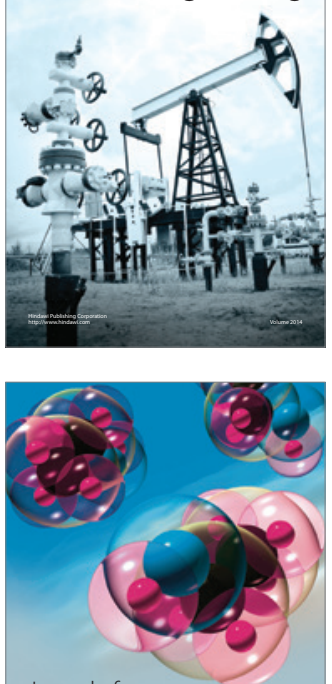

Fuels
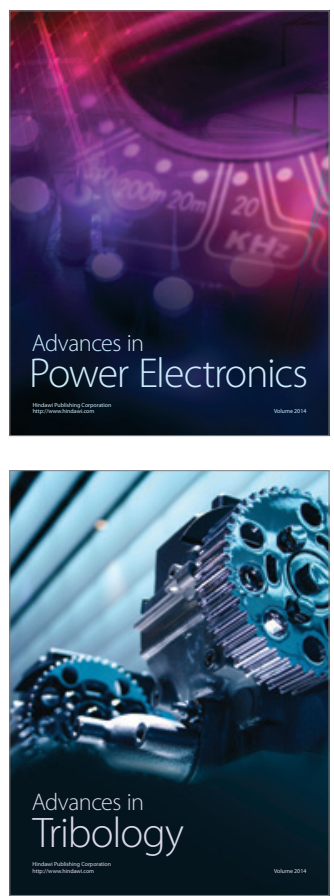

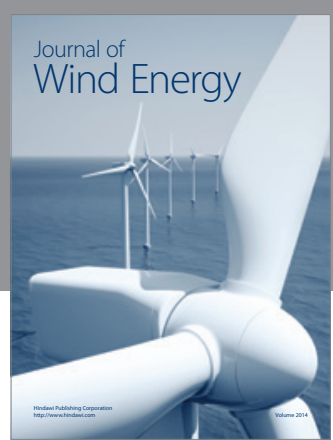

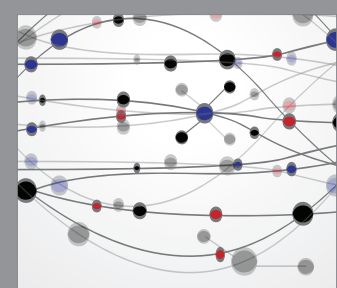

The Scientific World Journal

Submit your manuscripts at http://www.hindawi.com

Journal of

Structures
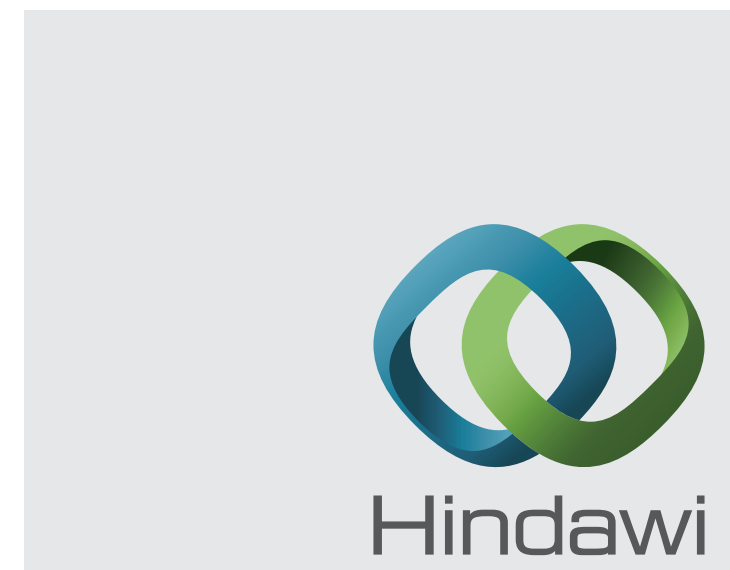

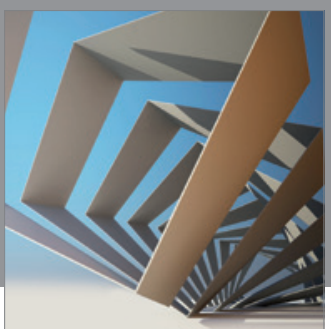

Rotating

Machinery
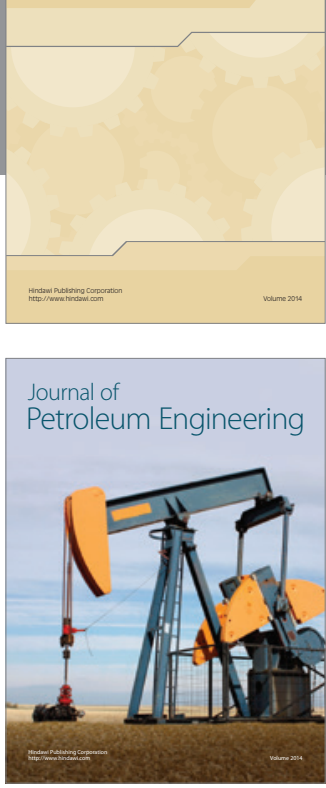

Journal of

Solar Energy
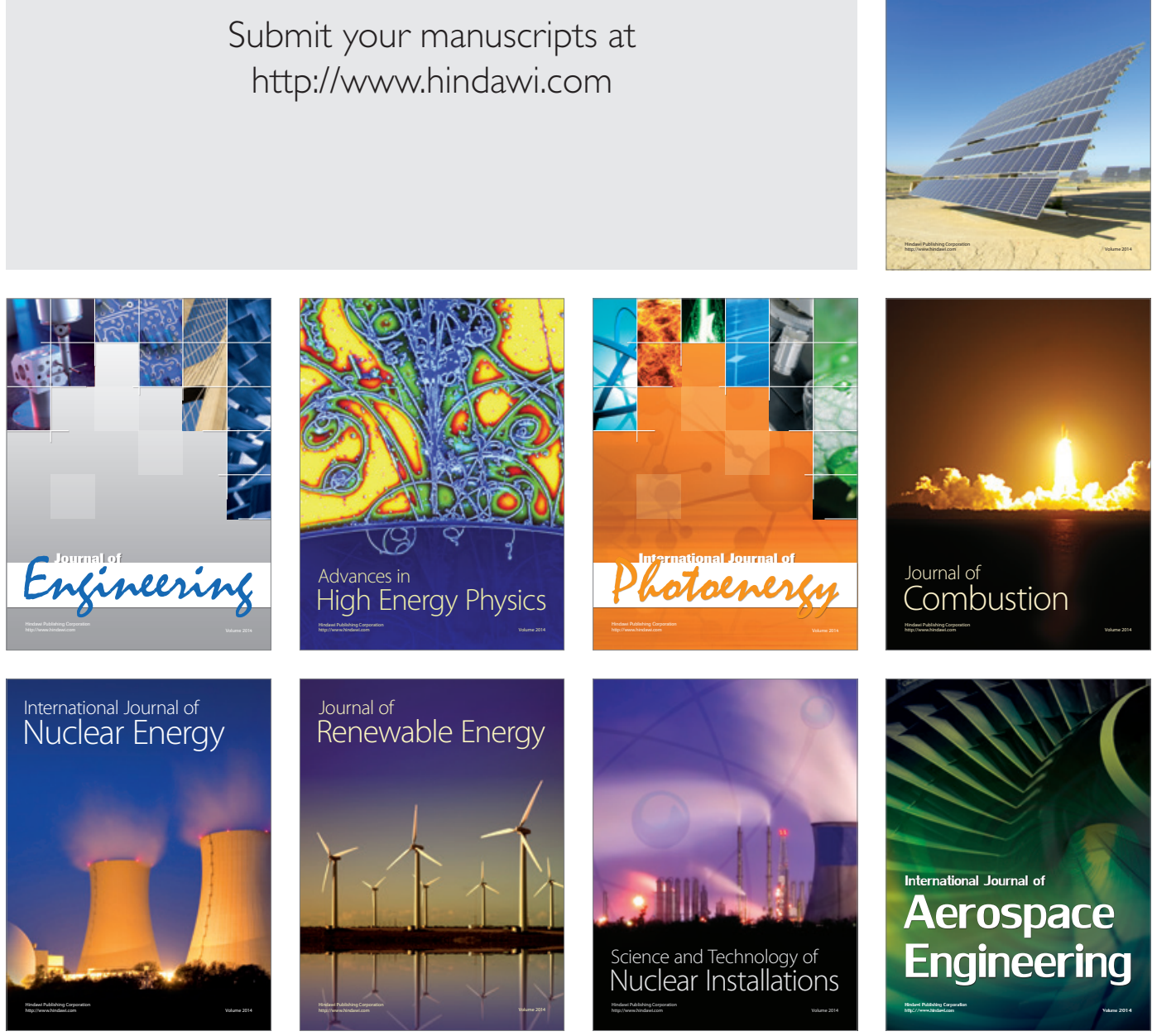